Lwoff, A. (1957). J. gen. Microbiol. 17, 239-253

\title{
The Concept of Virus
}

\section{The Third Marjory Stephenson Memorial Lecture}

\author{
BY A. LWOFF \\ Institut Pasteur, Paris
}

Things thought too long can be no longer thought, For beauty dies of beauty, worth of worth, And ancient lineaments are blotted out.

William Butler Yeats: The Gyres (Last Poems, 1936-39)

From the letter inviting me to give this third Marjory Stephenson Memorial Lecture I understood that the subject should be 'general'. Most scientists, as you know, are maniacs. One of my manias is to consult dictionaries. The dictionary says that general means 'completely or approximately universal', but it fortunately adds 'within implied limits'. I decided that the limits of universality would be the borderline of the kingdom of viruses.

The notion of virus, the subject of this lecture, is a pons asinorum of microbiologists, and I suspect that some of you have come to see how I behave on the asses' bridge. It seems therefore only fair that I should take the maximum of risks. Thus I have decided not to avoid discussing the notion of organism and the notion of life, which are considered to be highly treacherous subjects.

The man in the street generally considers viruses as the dangerous agents of infectious diseases. If one has to lecture before an assembly of microbiologists, one becomes aware of the fact that the real danger lies with the virologists. When by reading their writings one tries to understand what a virus is, one reaches a sort of feeling of the possible existence of some slight theoretical misunderstandings amongst virologists in which it may be dangerous to be involved. Some virologists are convinced that viruses are micro-organisms. This view is expressed in Sir MacFarlane Burnet's book Virus as Organism. Other virologists, like Wendell Stanley, feel that viruses should be considered as molecules. A third class is represented by F. C. Bawden and N. W. Pirie who write, 'statements that viruses are small organisms should be regarded with as much suspicion as statements that they are simply molecules'. If one wants to know who is right, one needs only to read Dr C. H. Andrewes's article Viruses as Organisms: 'It is my experience', writes Dr Andrewes, 'that those who have studied viruses from the widest point of view are more apt to consider them as organisms, whereas others who have looked on them from perhaps a more restricted point of view are more apt to toy with other hypotheses.'

In the light of these remarks the negative conclusion of Bawden \& Pirie might appear as the result of the absence of any view, whether wide or 
restricted. Being however in complete agreement with them, I am inclined to consider that this opinion is the result of concentrated thinking.

If a virus be neither organism nor molecule, what is its nature? What is a virus? It is a malady of our time that words are often deprived of their meaning. Many people like to think that a virus is something different from a virus.

My ambition is to show that the word virus has a meaning, and I shall defend a paradoxical viewpoint, namely that viruses are viruses. As simplistic as this statement might appear to some of you, the task is not an easy one. It will be necessary to analyse critically the behaviour and properties of viruses at various phases of their life cycle, and to find out the nature of the difference between viruses and other infectious agents, between viruses and micro-organisms. Then, if we have landed somewhere, we have to decide where we have landed. A brief historical survey is here a necessity.

\section{History}

Until the end of the nineteenth century the history of viruses is just a part of the history of infectious diseases. In about 2500 B.c. the Chinese had identified small-pox and knew that it was transmissible. To write however without comment, as some people do, that the Chinese knew viral diseases is perhaps not entirely justified. Aristotle was aware of the fact that rabies was transmitted by the bite of dogs, and the Hebrews used to compare this bite to that of a venomous snake. In latin virus means 'venom' or similar poisonous fluid. A virus was something which could produce a disease. And in A.D. 50, Cornelius Aulus Celsus produced this remarkable sentence: 'Rabies is caused by a virus.' The ideas of Celsus about viruses, considered in the light of our present knowledge, were probably rather primitive and therefore Celsus should not be considered as the real founder of virology.

Ideas concerning infectious diseases remained metaphysical until the concept of a specific agent emerged. This was mainly the result of the study of epidemics of syphilis which in this respect, as well as in the development of antibacterial chemotherapy, has been a highly beneficial disease.

Then, mainly as a consequence of Pasteur's work, the agents of infectious diseases were identified as microbes. These agents whether bacteria, protozoa or fungi, were called viruses.

Pasteur and Roux had no difficulty in proving that rabies was a specific infectious disease. Although they were unable to see the agent, they quite naturally considered it to be a small microbe. When Iwanowsky discovered that the juice of tobacco plants showing the symptoms of mosaic disease remained infectious after filtration, he also concluded that the infectious agent was a small microbe. Then came Beijerinck who confirmed the filterability of tobacco mosaic virus. He also discovered that the infectious power was not lost by precipitation with ethanol and that the infectious agent could diffuse through agar gels. The infection, wrote Beijerinck, is not caused by microbes but by a fluid infectious principle. This intuition of genius about a difference of nature 
between tobacco mosaic virus and micro-organisms makes Beijerinck the real founder of conceptual virology.

Beijerinck's views were so opposed to the current ideas that they did not receive any attention. This did not prevent the discovery of a number of infectious filterable agents which were considered to be small microbes and which were therefore called filterable viruses or ultraviruses. Then someone remarked that, because ultraviruses were small microbes, they should be called inframicrobes. For obvious reasons, none of the scientists studying filterable infectious agents was pretentious or modest enough to describe himself as an ultra-virologist or as an infra-microbiologist. And as everybody has to be labelled, these scientists were labelled as virologists. Quite naturally, as a result of the principle of the least effort, the ultraviruses, the filterable invisible infectious agents studied by virologists, became viruses. And as a counter-stroke the microbes, the visible infectious agents, were deprived of their ancestral right to be called viruses. This swing produced a confusional state from which microbiology has not yet recovered. One of our problems will be to find out whether or not the disease is curable.

\section{The bacteriophage}

A general lecture should stand high above technical details and should therefore not include any data. The notion of virus, however general it may be, nevertheless has to be founded on a few facts. And I hope to be forgiven if I remind you briefly of the life cycle of a bacteriophage.

A bacteriophage is a nucleoproteinic particle adorned with a tail. When the tip of the tail meets a receptive bacterium it attaches itself to the bacterial cortex. Then the genetic material of the phage, that is to say its nucleic acid, passes into the bacterial cytoplasm. The infected bacterium can then evolve in two ways.

(1) The genetic material of the phage multiplies and specific phage proteins are synthesized. This is the vegetative phase, at the end of which some 100 phage particles are organized. They will be liberated as a result of the lysis of the bacterium.

(2) The genetic material of the phage does not multiply. It reaches a specific locus or receptor of the bacterial chromosome and attaches itself to it. By virtue of this attachment, the properties of the genetic material of the phage are modified and it is now a prophage. The prophage behaves as if it were a bacterial gene. It divides together with the bacterial chromosome and at the division is transmitted to each of the daughter bacteria. From time to time, either for unknown reasons or as the result of the action of mutageniccarcinogenic agents, the prophage loses its connexion with the chromosome, starts the vegetative phase of the cycle, and phage particles are produced. Bacteria which carry a prophage are called lysogenic. They are endowed with the property of producing phage in the absence of infection. The prophage is the substratum of this property. It is the structure which carries the information necessary for the production of phage particles. 
We have now to discuss a few features and properties of bacteriophage. As rightly emphasized by Luria, the ability to be introduced from outside is a requirement for the recognition of a virus as such. This is, by the way, one of the few theoretical points on which all virologists agree. If infectiousness is eliminated from the definition of viruses then we are no longer able to discriminate between viruses and the cellular organelles endowed with genetic continuity. Now we can perform a test and ask a few questions of a certain number of people.

\section{Table 1. The temperate phage}

The genetic material of the phage or germ may be in three states: proviral genome; vegetative genome; infective genome.

Phase of the

life cycle

Proviral

Vegetative

Infective
Constitution

Nucleic acid (NA); Replication of

nucleic acid

Nucleic acid $+\quad$ Replication of proteins of phage; nucleic acid.

Synthesis of phage proteins

Nucleoprotein ;

$\begin{array}{cc}\text { Pathogenicity } \\ \text { Infectivity } & \text { as such }\end{array}$

$\mathbf{0}$

0

$\mathbf{0}$

$+$

$+$

If one asks whether the prophage or the vegetative phage is a virus, the answer is no. If one asks whether the phage particle is a virus, the answer is yes. The reason is simple. Infectivity being considered as a characteristic trait of viruses, something is recognized as a virus only when it possesses this feature. And we deny the right of being called viruses to those phases of the life cycle which are devoid of this character. This is exactly as if one would deny the right of being a Plasmodium to those phases of the life cycle of the malarial parasite which are not infectious for the mammal. Allow me here a short digression.

Mammals are said to be characterized by the possession of mammae which secrete milk for the nourishment of the young. And it is true that mammals produce milk. It is also true that a number of us who will never produce any milk are nevertheless mammals. This remark might seem irrelevant. It has however a profound meaning which reaches far beyond mammals and viruses. It means that the most distinctive characteristics of a class are not necessarily present, are not necessarily expressed, in all the individual members of the class at each phase of their life cycle.

In more general terms it means that the properties of a category of a given order are transcended by the properties of a category of a higher order. This is of the utmost importance when discussing the definition of a phage. Reproduction is a necessary feature of bacteriophage. If one denies to the vegetative phage the right to be called a phage because it is not infectious, one should not call the bacteriophage particle a virus because it does not reproduce itself. This is obviously absurd.

It is evident that a bacteriophage is neither this nor that but is necessarily the sum of the various phases of its life cycle. Any definition of a bacterio- 
phage should be an integration. When one considers the life cycle, one sees first that the only structure which is common to all the three phases is the genetic material. One sees also that the infectious phage particle never multiplies as such, but is produced by the organization of phage material. An infectious particle is never the direct descendant of another infectious particle. Ultimately, as already seen, some of the phases of the cycle are not infectious.

Table 2. The bacteriophage as a whole

Specific nucleic acid

Replication of nucleic acid

Synthesis of proteins; pathogenicity;

morphogenesis of particles

Infectivity

$\begin{array}{ccc}\text { Prophage } & \begin{array}{c}\text { Vegetative } \\ \text { phage }\end{array} & \begin{array}{c}\text { Phage } \\ \text { particle }\end{array} \\ + & + & + \\ + & + & 0 \\ 0 & + & 0 \\ & & +\end{array}$

A definition of the phage should therefore not be centred on the infectious particle. The following definition is proposed: a bacteriophage is a strict parasite of bacteria possessing an infectious phase and which is multiplied in the form of its genetic material and which is sometimes pathogenic. According to this definition, the prophage and the vegetative phage are parts of the 'bacteriophage' as well as the infectious particle which is thus deprived of its supremacy.

The notion of bacteriophage should not be transcended by the notions of infection and of disease, which are only particular aspects of the bacteriophage considered as a whole.

\section{Infection}

By considering phage with more attention the conclusion will be reached that it is not only the supremacy of the infectious particle which is on trial but the notion of infection itself.

According to the Oxford Dictionary, infectious means: having the quality or power of communicating disease by infection. And infection is defined as the communication of a disease. Let us consider a few examples.

A typhoid bacillus infects an animal which dies of typhoid. The agent of the disease is the infectious bacterium. There is no problem. If one injects spores of tetanus bacilli into an animal, the spore is infectious, but the active agent is the bacterium which is inside the spore. A mosquito carrying Plasmodium malariae gives malaria to an animal. The mosquito is infectious, but it is clear that the agent of the disease is not the mosquito, but the malarial parasite.

A bacteriophage particle injects its genetic material into a bacterium which dies. The phage particle is infectious, but the agent of the disease is the genetic material of the phage which has started the vegetative phase. A male lysogenic bacterium copulates with a non-lysogenic female. The male bacterium injects its chromosome and its prophage into the female which dies. The male lysogenic bacterium is infectious, but the agent of the disease is the prophage. Thus, the genetic material of the phage, which is by itself not infectious, is the 
agent of the infectious disease. What is more disturbing is that the penetration of the genetic material of the phage is not necessarily followed by a disease. The bacterium can be lysogenized as the result of an 'infection' and remain healthy. Should the penetration of the genetic material be described as an infection or not depending on the fate of the bacterium?

When scrutinizing all the cases of 'infection' it becomes manifest that the essence of infection is not the disease, but the introduction into an organism of a foreign entity able to multiply, to produce a disease, and to reproduce infectious entities.

This definition eliminates all the pitfalls and excludes from infectiousness all the normal cell structures which can penetrate into another cell, such as the transforming principle, the lethal genes, and the gene which controls the synthesis of bacteriocins. It is clear that one has to discriminate between the thing which infects or injects and the object which is injected. The foreign entity, which penetrates into, and reproduces in, an organism as a result of an infection is a sort of infectum. When dealing with bacteria, the infectum is the bacterium itself. When dealing with bacteriophage, the infectum is its genetic material.

This reasoning will probably be considered as specious and sophisticated and I shall be accused of rigorism, as if strictness were not simply exactitude and precision.

It is well known that we are unable to think without mental schemes. It is therefore better to know what these schemes are. Because bacteriophage is the agent of an infectious disease, we have applied to it the scheme and terminology derived from the study of infectious diseases caused by microorganisms, and, as a result, our ideas have been distorted.

\section{Viruses in general}

So far, we have considered bacteriophage as a model of virus. We should now consider viruses in general. An important remark is necessary here. Mammals are defined as animals able to secrete milk. It appears immediately that this definition has a value only if other animals exist which do not secrete milk. Whatever the importance of a class, its definition must not only include some objects, but exclude other objects. The presence of a character as a part of the definition of a class has its necessary counterpart in its correlative absence in another class. The concept of mammals has a meaning because of the existence of animals such as reptiles or birds which do not secrete milk, which are not mammals.

We speak of viruses as different from bacteria, protozoa, fungi and algae. This implies the existence of a category of infectious agents, viruses, which are different from the other infectious agents. As we aim to be scientists, we have to state clearly the nature of the difference.

Viruses are often opposed to bacteria because of their size. A virus, according to some virologists, should have at least one dimension lower than $200 \mathrm{~m} \mu$. If dimensions have any meaning it is not by the astrological virtue of a 
number, but because of a correlation between size and some essential properties which are responsible for fundamental differences.

In order to find out the essence of this difference, let us examine and compare the bacteriophage considered as a model of virus on the one hand, and on the other hand, a typical micro-organism such as protozoon, a yeast or a bacterium.

(1) All typical micro-organisms contain both types of nucleic acids, deoxyribonucleic acid (DNA) and ribonucleic acid (RNA). Bacteriophage contains only one type, DNA.

Table 3. Organisms, viruses and cellular constituents

Types of nucleic acid

Multiplying as nucleic acid and

produced from nucleic acid only

Growth and division*

Presence of Lipmann system

Infectivity

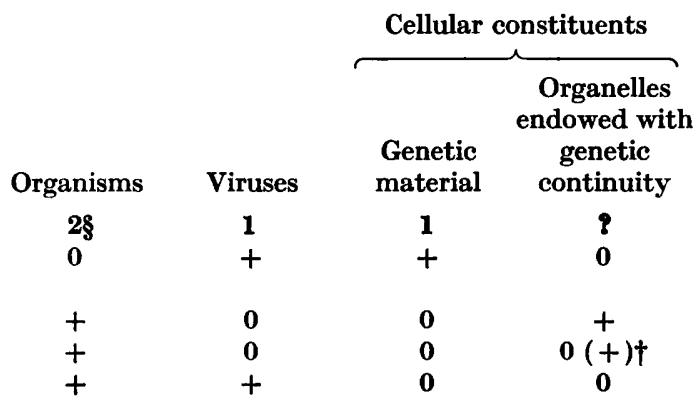

* Concerns micro-organisms only. The replication of a structure by the template mechanism is neither considered as growth nor as division but as replication.

$\dagger$ Some organelles only contain a Lipmann system.

$\S 2=$ DNA + RNA, $1=$ DNA or RNA.

(2) All typical micro-organisms are reproduced from the integrated sum of their constituent parts, nucleus, cytoplasm, cytoplasmic structures, cortex. Bacteriophage is produced or reproduced from its nucleic acid only. When considering a micro-organism, the infectum is the micro-organism itself; when considering a bacteriophage, the infectum is its genetic material.

(3) The essence of the multiplication of any cell or micro-organism is the replication of the genetic material, but multiplication is not separable from growth which is the result of the synthesis of cell material. During the growth of a micro-organism, the individuality of the whole is maintained. Growth culminates in binary fission. Binary fission has no meaning at the molecular level. An individual molecule cannot undergo fission; the replication of nucleic acid by a template mechanism is not a binary fission. Binary fission has a meaning only when we consider either a complex organelle or structure or an organism.

The essence of phage multiplication is also the replication of its nucleic acid. During the vegetative phase, there is an increase of phage material, but the nucleic acid and the proteins, so far as we know, do not form an organized structure. What increases is a disorderly mixture of phage constituents. There is nothing which could divide. There is no binary fission in a bacteriophage.

(4) Micro-organisms possess a system of enzymes which convert the potential energy of foodstuffs into the high energy bonds which are needed 
for biological syntheses, including patternization. This system, which might be called the 'Lipmann system', is part of the micro-organism itself. The Lipmann system is absent from bacteriophage. The energy for the synthesis and patternization of phage material is provided by the host cell.

Numerous small infectious agents, whether they are parasites of plants or animals, possess a number of characters which are present in bacteriophage. They possess only one nucleic acid; they multiply in the form of their nucleic acid; they can be reproduced from their nucleic acid; they are unable to grow and to undergo binary fission; they depend for their energy on the Lipmann system of the host cell.

Viruses could accordingly be defined as: strictly intracellular and potentially pathogenic entities with an infectious phase, and (1) possessing only one type of nucleic acid, (2) multiplying in the form of their genetic material, (3) unable to grow and to undergo binary fission, (4) devoid of a Lipmann system. It should be noted that any one of characters 1 to 4 is sufficient to identify an entity possessing an infectious nuclear-proteinic phase as a virus, and it is quite possible that these features or attributes are correlated and subordinated.

If one wants to put emphasis on the infectious particles the following definition may be proposed: viruses are infectious, potentially pathogenic, nucleoproteinic entities possessing only one type of nucleic acid, which are reproduced from their genetic material, are unable to grow and to undergo binary fission, and are devoid of a Lipmann system.

As already seen, an object, a category or a concept, has an individuality or a reality only because of the existence of different objects, categories or concepts. The art of definition is founded on differences as well as on resemblances. A definition has to exclude as well as to include. Our definition of viruses is valid only because, at the same time, it includes a homogeneous class of entities, viruses, and excludes another homogeneous class of entities, microorganisms.

We are therefore justified in uniting into a special class those entities called viruses. The concept of virus is thus established on a firm ground and the term virus has, at last, a definite meaning.

\section{Are viruses organisms?}

Small infectious agents have been subdivided into two categories: viruses and non-viruses, the latter being micro-organisms. Micro-organisms are organisms. Are viruses organisms?

Let us make here another mammalian digression. Mammals are obviously not reptiles, but they have evolved from a primitive reptile. Thanks to this common origin, mammals share with reptiles a certain number of traits, as for example the presence of vertebrae. Therefore they are united into a group of vertebrates which transcends the differences and exemplifies what is in common.

If viruses have evolved from micro-organisms, whether primitive or not, they might have kept one or many features of organisms. The existence of 
those common features would allow one to visualize viruses as a special class of micro-organisms, that is to say, to visualize viruses as organisms.

The first thing to do is to define organisms. Micro-organisms being organisms, it is plain that all the main characters which have been ascribed to micro-organisms are among the characters of an organism. These are: the presence of two types of nucleic acids (DNA and RNA), the ability to grow, the presence of the Lipmann system. These features are also present in the cells of multicellular organisms, the cell stricto sensu. Since it is obvious that a cell stricto sensu is not an organism, these features are not sufficient to define an organism.

An organism may be visualized as an independent unit of integrated and interdependent structures and functions. In a multicellular organism, the integrated and interdependent parts are the cells. We shall leave these multicellular organisms aside and consider only micro-organisms or protista. In protista, the integrated and interdependent parts are the cellular organelles or structures, such as the chromosomes, the mitochondria, the enzymes, the cortex, etc. Cellular organelles are not organisms because they are dependent, because they are parts of a whole which is the organism itself, the ultimate unit of independent reproduction.

One of the main tasks of an organism is to control its dependent and interdependent parts. Harmonious growth is the result of the balanced synthesis of cellular material and the balanced replication of various structures. We know to-day that harmonious growth is controlled by the interplay of metabolites and antimetabolites produced as the result of enzymic activity. Let us then consider critically the arguments which are generally put forward in order to justify the statement that viruses are organisms.

(1) 'Viruses multiply; they are reproduced true to type; they contain nucleoproteins'.

These characters are actually to be found in organisms. Unfortunately, they are to be found, either isolated or together, in a number of cellular organelles such as the nuclei, the mitochondria, the chloroplasts, the kinetosomes.

(2) 'Viruses are antigenically different from the host cell'.

In a cell each individual enzyme has its antigenic individuality. Such a thing as antigenicity of a cell in general does not exist.

(3) 'Viruses and bacteria form a regular gradient of size.'

This is true, but between a molecule and an elephant one finds entities of intermediary sizes and this does not prove that an elephant is a molecule or vice versa.

The rules of formal logic establish the validity of a syllogism. These rules have to be applied by virologists. In order that a character should be valid for the union of two classes into a common category, this character should be absent from other categories. For example, worms and vertebrates possess a digestive tract, but this does not prove that worms are vertebrates because insects also possess a digestive tract. Viruses and organisms have a few characters in common. These characters being also present in cellular 
organelles, they cannot be considered as supporting the conclusion that viruses are organisms. It is really strange that so many people take delight in looking straightforwardly at problems which they have so carefully posed askew.

Almost sixty years ago Beijerinck pointed out that the multiplication of tobacco mosaic virus has many points in common with the multiplication of amyloplasts and chloroplasts. Despite the fact, said Beijerinck, that both the virus and the plastids have an independent existence, their multiplication is controlled by cellular growth; to-day we would rather say by cellular metabolism. Bawden has reached the conclusion that: 'If we are to seek analogues for viruses, the closest may well lie among cellular components'. When one considers the essential properties of viruses it is clear that they have more in common with cellular organelles than with micro-organisms.

In a general article on the influenza virus which appeared in February 1957, one may read the following: 'a virus is not an individual organism in the ordinary sense of the term, but something which could almost be called a stream of biological patterns'. I should like to say that I am in complete agreement with this statement which, by the way, is due to Sir MacFarlane Burnet.

\section{Are viruses alive?}

The classical question as to whether viruses are or are not alive depends necessarily on our conception of life. Life may be considered as a property, a manifestation or a state of cells or organisms. This excludes from the living, all cellular particles or structures, whether or not endowed with genetic continuity. It is evident, as already seen, that the properties of an organism are more than the sum of the individual properties of its individual parts. An organism is the result of the integration of its dependent and interdependent parts. The essential character of an organism, independence, with all its implications, transcends the characters of its parts, dependence. Life is precisely this transcendence. Those who share this viewpoint will conclude that viruses are not alive.

\section{The origin of viruses}

Most of those who have discussed the problem of the origin of viruses have considered the virus particle. Let us instead consider briefly the problem of the origin of the prophage.

Two main theories have been proposed: (1) the prophage is the residue of the degradation of a parasitic bacterium or of a more or less primitive organism; (2) the prophage was born by genic or chromosomal mutations of the bacterium which thus became lysogenic. Instead of trying to find which theory is right, let us try to build a third theory which would suppress the contradiction.

Bacteria are a very homogeneous and certainly monophyletic group, which differ from all other protista by the organization of the mitochondrial apparatus. This means that Escherichia coli, for example, and the hypothetical parasitic bacterium which is supposed to have degenerated into a prophage of $E$. coli have a common ancestor. In other terms the genetic material of $\boldsymbol{E}$. coli and the genetic material of the prophage have originated from the very same 
genetic material. And the common structure which has been preserved in the bacterial chromosome as a receptor, and in the prophage where it accounts for lysogenization, is the signature of the common origin.

If one admits that the prophage had its origin in the genetic material of a bacterium, it might have originated in any bacterium and especially in a more or less remote ancestor of the very bacterium which is now lysogenic. The phase at which evolution has taken place is not as important as the existence of a common origin. The endogeneous and exogeneous theories can be reconciled in the following way: the genetic material of the bacteriophage and the genetic material of the bacterium have evolved from a common structure, the genetic material of a primitive bacterium. Whatever the origin of the genetic material of the bacteriophage might have been, we know to-day that the prophage behaves as if it were a bacterial gene: its reproduction is controlled by the bacterium as a whole just as is the reproduction of any bacterial gene or bacterial structure. The prophage is obviously not independent. It behaves as a dependent part of an organism, but this organism is not the bacteriophage, it is the bacterium.

Between 1855 and 1870 , microbiology was founded. It was established that micro-organisms were specific living beings which always came from an identical living being. An animal injected with a specific microbe shows a specific disease and the specific infective agent is reproduced true to type. This is true for bacteria and also for viruses. The theory of spontaneous generation or heterogenesis according to which a living organism could arise from anything or everything was wiped out.

A theory is a generalization and it tends to absorb as much as possible, especially those aberrant data which, in the purgatory of science, await an interpretation. The more truth a theory contains, the more it includes. The more it includes, the greater its power. The greater its power, the more dangerous it is. A discovery leads to a theory, a theory to a law, a law to a religion. According to the dogma, infectious diseases are caused by specific micro-organisms. Viral diseases being infectious, it was concluded that viruses were specific micro-organisms. And when the idea was proposed that viruses were perhaps not microbes but might have originated from some pathological constituents of their host cell, then the theory of spontaneous generation seemed to revive from its ashes. The supporters of the endogeneous theory were accused of heresy and a smell of sulphur was floating in the air. The inquisitors of faith have tried, and are still trying, to ridicule the endogenous theory by brandishing the threadbare scarecrow of heterogenesis. The endogenous theory of the origin of viruses is a theory. It may be wrong, but it is not absurd. If discussed at all, it should be discussed scientifically.

Those who claim to possess the truth should remember that heterogenesis was once 'the truth'. And the spectre of heterogenesis should be allowed to rest in the famed purple sheet where scientists shroud their dead gods.

When one walks in the country one sees cows, sheep, hogs, horses, men, one 
sees individual mammals. One never sees a mammal in general because such a thing does not exist. The mammal in general could be a dream, but it is a

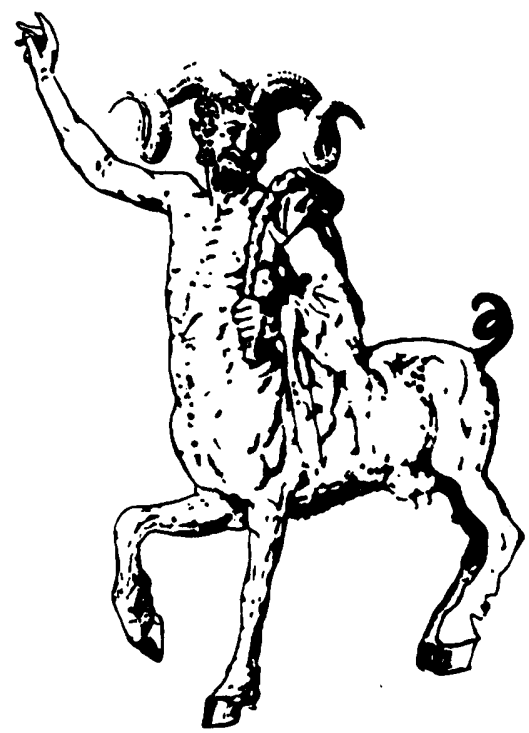

The mammal in general could be a dream....

concept. And it is essential to remember that a concept is an idea of a class of objects. The virus is a concept. Our ideas concerning the origin of viruses may differ, and also our ideas of the place we assign to viruses among other entities. The very use of the term virus, however, implies the acceptance of viruses as a specific class of entities. The choice of a discriminative character of a class cannot be a matter of taste. Either a character offers itself indiscriminately to any category and is valueless, or it belongs exclusively to one category and is thereby discriminative, that is to say valid. A concept is the equivalent of a doctrinal corpus. It has to be sound and coherent and should stand firm on a strictly logical base.

\section{Autocriticism}

Whilst giving birth in pains and tears to this concept of the virus I have kept a diary. It was a sort of analysis and criticism of the intellectual operations involved in the process. Autocriticism being nowadays rather fashionable I thought an abridged version of the diary would not be completely out of place as a manner of conclusion. Those who might feel sad at the idea of being deprived of the pleasure of heterocriticism may be reassured at once: I have left aside for their personal enjoyment all the really critical points.

So, what have I done? The data pertaining to viruses have been considered in themselves and then integrated, that is to say, united in order to form a whole. To cement the stones a mental element was introduced. The operation which has been performed is what Whewell called a colligation, a colligation 
being defined by the Master of Trinity as 'an operation by which facts are united under one and the same idea'. The word was new at the beginning of the nineteenth century, but the idea was an old one. It corresponds to the Aristotelian transition from particular to general, the transition to a superior degree of generality. It is closely related to Bacon's Interpretatio naturae, and also to induction which is the transition from facts to laws. I have not dealt with laws, but I have attempted to visualize the virus as a whole, to introduce a general idea, the notion or concept of virus. In doing so, I have tried to be as rationalistic and logical as possible.

I have been constantly aware of the fact that I had to submit my thoughts to the compatriots of John Stewart Mill whose empirical logic has exerted and still exerts a considerable influence in this country. It is customary to oppose British empiricism to French logic. Let us see what happens sometimes on the continent.

In his Introduction à l'Etude de la Médecine Expérimentale, Claude Bernard claimed that philosophers were without utility for scientists; that they loaded the mind of scientists with all sorts of inapplicable principles. The useful principles, said Claude Bernard, are those which emerge from the details of experimental practice. Claude Bernard, who was neither an empiricist nor a logician, may be designated as an experimentalist. And perhaps, in the last analysis, the difference between empiricism and logicism is not so marked in reality as in the brains of philosophers. It would seem that a number of scientists, although empiricists, produce perfectly valid concepts. This is really depressing, and philosophers cannot do much about these aberrant people except to describe them as unconscious colligators.

'Frenchmen', said Paul Valéry, 'deem possible, and even in conformity with the essence of things, that a prodigiously diverse ensemble of highly complex phenomena can be and must be condensed and finally reduced into a few plain formulae, at the same time necessary and sufficient.' Belonging to an hyperlogical extrovert nation, I have coined numerous definitions as if I had really penetrated the essence of things. And I should not have discussed the intimate nature of viruses with more confidence if $I$ had been myself a virus.

'The French', said T. E. Lawrence, 'see by the directly-thrown light of reason and understanding, not through the half-closed eyes, mistily, by things' essential radiance, in the manner of the imaginative British.'

Some scientists visualize the virus as an ill-defined shape emerging bashfully out of a dense and golden cloud. This is a beautiful and romantic vision. Virology should, however, not be too Turnerian. Nor should it be an abstract art. The portrait of a virus should not produce an aesthetic emotion by means of an organic disturbance. The virus is amenable to intellectual analysis. I have cut its life cycle into slices and analysed each slice as lucidly as I could. The significance and value of each character has been dissected with a sort of sadism. This, I realize, is mere intellectual butchery, the type of job which it is advisable to leave to somebody else. 
As a result, the virus has been unveiled. Now, it stands before you, naked. Do not turn your eyes away, it is only a concept. And the concept of virus, just as any other concept, the concept of a lecture, the concept of woman, is not as difficult to handle as the real object.

I have tried to make everything as clear, as perceptible and as ostensible as possible. The operations which have been performed were required for intellectual hygiene and intellectual comfort. And yet, I am afraid that in performing this task $\mathrm{I}$ have deprived myself of one of the most prized privileges of elegance, namely invisibility.

According to William James, to ignore, to disdain, to consider, to overlook, is the essence of the gentleman. I have considered, but I have not ignored, nor disdained, nor overlooked enough. Perhaps I have displayed a truly plebeian excess of logic and of passion. It would perhaps have been more aristocratic to look at things from a distance with a haughty and stylish detachment.

It is certainly pleasant to be a mammal. Yet, I am conscious of having produced such an excessive number of mammalian digressions that you might have had the impression that the lecture was dealing with the concept of mammal rather than with the concept of virus. Also, I have made use of the American word patternization. Mea culpa. Mea culpa. I confess my sins and hope they will be remitted. I have behaved improperly and as a punishment, the conclusion of this lecture will be prosy, coarse and vulgar: viruses should be considered as viruses because viruses are viruses.

\section{APPENDIX}

\section{Remarks on the pathogenicity of viruses}

Viruses are pathogenic or potentially pathogenic entities. The property of eliciting a disease is shared by a number of micro-organisms and by a few abnormal cellular organelles such as, for example, lethal genes. Bacteria, just as 'higher organisms', also exhibit diseases of metabolism. It is known, for example, that the synthesis of colicin, pyocin, megacin or in more general terms, of bacteriocins, is a fatal disease. These lethal biosyntheses are, so far as we know, controlled by genetic determinants. The originality of virus is that the malady initiated by the genetic material generally culminates in the morphogenesis of infectious particles.

The objection will naturally be raised that oncogenic viruses which are responsible for malignant growth do not kill their host cell. This is true, yet the oncogenic virus kills the organism. When one considers the pathogenic action of an ordinary virus such as bacteriophage, one sees that the virus kills its host cell because it disturbs the equilibrium of its dependent parts. If one analyses the pathogenic action of oncogenic viruses one realizes that they modify the host cell which is a dependent part of an organism. In general terms, when considering the organism which transcends its individual interdependent parts, we have the right to say that oncogenic viruses are pathogenic. 


\section{REFERENCES}

Andrewes, C. H. (1952). Viruses as organisms. In Poliomyelitis. Second int. Poliomyelitis Conf. pp. 3-5. Lippincott Co.

BAwDEN, F. C. (1952). Virus and its interaction with the host cell: biochemical aspects. In Poliomyelitis. Second int. Poliomyelitis Conf. pp. 9-12. Lippincott Co.

Bawden, F. C. \& Pirie, N. W. (1951). Virus multiplication considered as a form of protein synthesis. In The Nature of Virus Multiplication, Symp. Soc. gen. Microbiol. 2, 21.

BeiJerinck, M. W. (1898). Über ein Contagium vivum fluidum als Ursache der Fleckenkrankheit der Tabaksblätter. Verh. Akad. Wet. Amst. 6, fas. 5.

Burnet, F. M. (1950). Virus as Organism. Harvard University Press.

Burnet, F. M. (1957). The structure of the influenza virus. Sci. Amer. 196, 37.

JACOB, F. \& Wollman, E. L. (1957). Genetic aspects of lysogeny. In The Chemical Basis of Heredity, p. 468. Edited by W. D. McElroy \& H. B. Glass. Baltimore: The Johns Hopkins Press.

Luria, S. E. (1953). General Virology. New York: John Wiley and Sons.

Lwoff, A. (1953). Lysogeny. Bact. Rev. 17, 269.

Lwoff, A. (1954). Control and Interrelations of Metabolic and Viral Diseases of Bacteria. The Harvey Lectures, series L, Academic Press, 92.

Lwoff, A. (1957). Cell and Organism. Proc. N.Y. Acad. Sci. 5, (in the Press).

Lwoff, A. (1957). Nutrition and metabolism in collateral fields. Cancer Res. (in the Press).

Stanley, W. M. (1952). Viruses as chemical agents. In Poliomyelitis, pp. 6-8. Second int. Poliomyelitis Conf. Lippincott Co.

(Delivered before the Society for General Microbiology at its Twenty-fourth Meeting, 15 April 1957) 